\title{
Kinematic analysis of a rolling tensegrity structure with spatially curved members
}

\author{
Philipp Schorr • Enrique Roberto Carrillo Li • Tobias Kaufhold • \\ Jorge Antonio Rodríguez Hernández • Lena Zentner • Klaus Zimmermann • \\ Valter Böhm
}

Received: 2 March 2020 / Accepted: 22 June 2020/Published online: 14 July 2020

(C) The Author(s) 2020

\begin{abstract}
In this work, a tensegrity structure with spatially curved members is applied as rolling locomotion system. The actuation of the structure allows a variation of the originally cylindrical shape to a conical shape. Moreover, the structure is equipped with internal movable masses to control the position of the center of mass of the structure. To control the locomotion system a reliable actuation strategy is required. Therefore, the kinematics of the system considering the nonholonomic constraints are derived in this paper. Based on the resulting insight in the locomotion behavior a feasible actuation strategy is designed to control the trajectory of the system. To verify this approach kinematic analyses are evaluated numerically. The simulation data confirm the path
\end{abstract}

P. Schorr $(\bowtie) \cdot$ T. Kaufhold · L. Zentner .

K. Zimmermann

Ilmenau University of Technology, Max-Planck-Ring 12,

98693 Ilmenau, Germany

e-mail: philipp.schorr@tu-ilmenau.de

T. Kaufhold

e-mail: tobias.kaufhold@tu-ilmenau.de

L. Zentner

e-mail: lena.zentner@tu-ilmenau.de

K. Zimmermann

e-mail: klaus.zimmermann@tu-ilmenau.de

E. R. C. Li · J. A. R. Hernández

Pontifical Catholic University of Peru, Av. Universitaria

cuadra 18, San Miguel, Lima, Peru

e-mail: ecarrillol@pucp.edu.pe following due to an appropriate shape change of the tensegrity structure. Thus, this system enables a twodimensional rolling locomotion.

Keywords Tensegrity structure $\cdot$ Inverse kinematics $\cdot$ Nonholonomic mechanics

\section{Introduction}

Tensegrity structures represent a special kind of prestressed frameworks consisting of one-dimensional members [1]. Due to the prestress, these structures enable free-standing self-stable equilibrium configu-

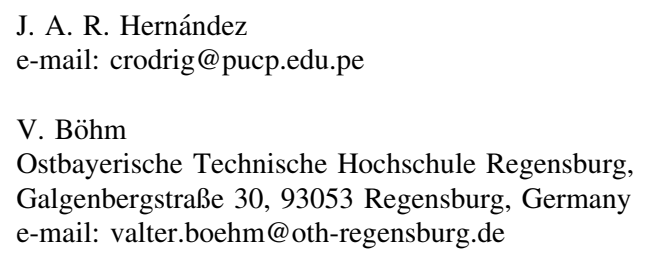


rations. According to the load at the equilibrium configuration, the members are classified as compressed members and tensioned members. The tensegrity principle requires that all compressed members are connected to each other only by tensioned members. Although tensegrity structures were originated in the fields of architecture [2] and modern arts [3] they recently accessed the fields of engineering due to their advantageous properties like shock resistance and great shape change ability. Therefore, engineers extended the definition of tensegrity. Hence, also curved compressed members can be applied [4] and only groups of compressed members have to be connected to each other by tensioned members [5]. Especially, in the fields of mobile robotics tensegrity structures are established. Various locomotion systems based on such structures are given in [6-9]. In particular, the rolling locomotion seems to be a appropriate approach to realize a controllable locomotion as shown in [10-12]. The shape of the structure is controlled by varying the prestress state. As a consequence of this actuation strategy the position of the center of mass is changing. Reaching a critical configuration a tilting motion is occurring. This tilting sequence is repeated successively to realize a desired locomotion. However, the tilting of the structure results in an impact. A real rolling locomotion system with precurved members is presented is [13]. The locomotion is controlled by the movement of internal masses to influence the position of center of mass. However, for a general two-dimensional locomotion additional tilting sequences are necessary. In [14] an approach of a rolling locomotion system based on a tensegrity structure, which allows a two-dimensional locomotion by pure rolling is investigated. The cylindrical shape of the locomotion system can be varied to a conical shape by controlling the prestress state.

Based on [14] a tensegrity structure with spatially curved members is applied as rolling locomotion system in this work. The use of a topology with only few members is targeted to guarantee a simple design of the locomotion system. The kinematics of the system are derived and a feasible actuation strategy to control the locomotion behavior is designed. Finally, numerical simulations are evaluated to verify the theoretical approach. The results confirm the occurrence of a controllable two-dimensional rolling locomotion due to a suitable shape change of the tensegrity structure.

In Sect. 2 the investigated tensegrity structure is presented. A mechanical model of the structure is developed and the kinematics of the rolling locomotion considering the nonholonomic constraints are derived. The locomotion behavior of the system is investigated in Sect. 3 focusing on the rectilinear locomotion, the circular locomotion and the general locomotion on a horizontal plane. In Sect. 4 a novel actuation strategy to follow a desired trajectory is derived. Furthermore, simulations are evaluated in order validate the actuation strategy. In Sect. 5 the results are concluded and an outlook for further investigations is given.

\section{Mechanical modeling of the rolling locomotion system}

\subsection{Topology of the tensegrity structure}

The tensegrity simplex shown in Fig. 1a represents the simplest three-dimensional tensegrity structure. This structure consists of three struts, which are connected by 9 cables. The state depicted in Fig. 1a is a stable equilibrium configuration. With regard to the load in this configuration the struts are compressed members and the cables are classified as tensioned members. The replacement of the straight struts of the simplex by spatially precurved members yields a cylindrically shaped tensegrity structure as shown in Fig. 1b. The connectivity of the members is modified in a small manner regarding to original tensegrity simplex. Due to the spatial precurvature of the struts, there is a multidimensional stress state. Nevertheless, following the common terms in the field of tensegrity structures, these precurved struts are declared as compressed members.

To verify the occurrence of a free-standing selfstable equilibrium configuration a demonstrator of the varied tensegrity simplex is developed. This demonstrator is depicted in Fig. 1c. The precurved compressed members are made of aluminum and the tensionend members are realized by steel wire. Indeed, a stable equilibrium state occurs and the shape is described by cylinder with radius $R=0.040 \mathrm{~m}$ and height $H=0.150 \mathrm{~m}$. 


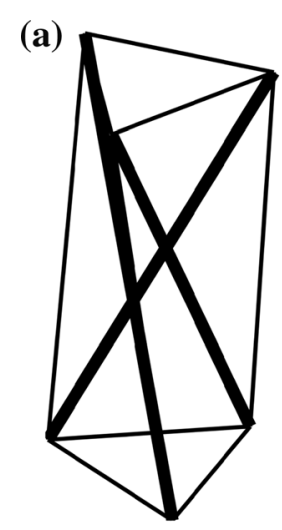

(b)

(c)

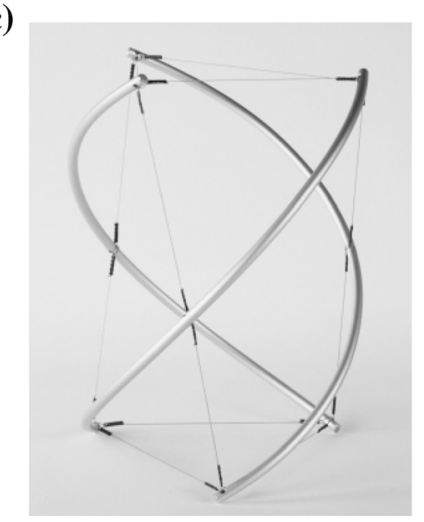

Fig. 1 Development of a rolling locomotion system based on a tensegrity structure-a simplex tensegrity (thick lines: compressed members, thin lines: tensioned members), b replacement of the straight struts by spatially precurved members, c real demonstrator of the tensegrity simplex with spatially precurved compressed members

Obviously, a cylinder features only a uniaxial bidirectional rolling locomotion. Thus, in order to realize a two-dimensional rolling locomotion a shape change of the tensegrity structure is required. This issue is realized by varying the length of selected tensioned members. Due to this actuation, the cylindrical geometry can be influenced to a conical shape which yields a circular rolling locomotion. The radius of curvature of the resulting trajectory depends on the conicity of the geometry. Finally, the combination of the rectilinear locomotion and the circular locomotion allows a general two-dimensional locomotion based on pure rolling. The actuation principle of the tensegrity structure to control the outer shape is shown in Fig. 2. The drive to realize the rolling of the system can be realized by the movement of internal masses as presented in [13].
The shape of the compressed members $(j=1,2,3)$ is defined by the vector $\boldsymbol{\rho}_{P}$ parameterized respective to $\varphi\left(\varphi \in\left[0, \varphi_{\max }\right]\right.$ with $\left.\varphi_{\max }=4 / 3 \pi\right)$. This issue is formulated in (1). The parameter $\beta$ represents the cone angle of the structure. In this work, the complex deformation of the spirals is simplified as shown in (1) respective to the body fixed Cartesian coordinate system $\{\xi, \eta, \zeta, C\}$ (C represents the geometric center of the tensegrity structure). Hence, the height $H$ of the cylinder and the cone is identical. Due to the limited shape change the parameter $\beta$ can be varied within $\beta \in\left(-\arctan \left(\frac{2 R}{H}\right), \arctan \left(\frac{2 R}{H}\right)\right)$. Applying the parameters of the demonstrator in Fig. 1c, the parameter $\beta$ is limited approximately to $\pm 28^{\circ}$.

$$
\begin{gathered}
\boldsymbol{\rho}_{P}=\left(\begin{array}{c}
\frac{H}{4 \pi}(3 \varphi-2 \pi) \\
R(\varphi) \cos \left(\varphi+j \frac{2 \pi}{3}\right) \\
R(\varphi) \sin \left(\varphi+j \frac{2 \pi}{3}\right)
\end{array}\right) \quad(j=1,2,3) \\
\text { with } R(\varphi)=R-\frac{H}{4 \pi}(3 \varphi-2 \pi) \tan (\beta)
\end{gathered}
$$

This issue clarifies that the radius of the cone in the $\eta-\zeta$-plane $(\varphi=2 / 3 \pi)$ is constant and cannot be influenced by the parameter $\beta$.

\subsection{Kinematics of the rolling locomotion system}

The rolling locomotion system is represented by the considered tensegrity structure which is in contact with a horizontal rigid plane due to gravity as shown in
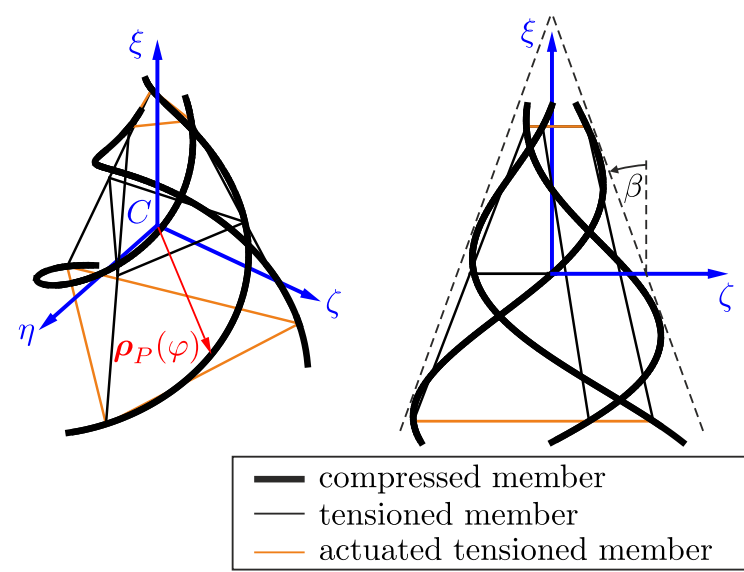

Fig. 2 Mechanical model of the considered tensegrity structure with conical shape due to the actuation 
Fig. 3. The position of the geometric center $C$ of the structure is described by the vector $\boldsymbol{r}_{C}=\left(x_{C}, y_{C}, z_{C}\right)^{\mathrm{T}}$ respective to an inertial Cartesian coordinate system $\{x, y, z, O\}$. The orientation of the tensegrity structure results due to three intrinsic rotations. This issue is given by a rotation respective to the $z$-axis of the inertial Cartesian coordinate system (rotation angle $\gamma$ ), a rotation respective to the resulting $y^{\prime}$-axis (rotation angle $\beta$ ) and a rotation respective to the resulting $x^{\prime \prime}$ axis (rotation angle $\alpha$ ). To transfer the vectors defined in the body fixed coordinate system $\{\xi, \eta, \zeta, C\}$ into the inertial coordinate system $\{x, y, z, O\}$ the transformation matrix $\mathbf{T}$ defined in (2) is applied.

$$
\begin{aligned}
& \mathbf{T}=\mathbf{T}_{\gamma} \cdot \mathbf{T}_{\beta} \cdot \mathbf{T}_{\alpha} \text { with } \mathbf{T}_{\alpha}=\left[\begin{array}{ccc}
1 & 0 & 0 \\
0 & \cos (\alpha) & -\sin (\alpha) \\
0 & \sin (\alpha) & \cos (\alpha)
\end{array}\right], \\
& \mathbf{T}_{\beta}=\left[\begin{array}{ccc}
\cos (\beta) & 0 & \sin (\beta) \\
0 & 1 & 0 \\
-\sin (\beta) & 0 & \cos (\beta)
\end{array}\right], \mathbf{T}_{\gamma}=\left[\begin{array}{ccc}
\cos (\gamma) & -\sin (\gamma) & 0 \\
\sin (\gamma) & \cos (\gamma) & 0 \\
0 & 0 & 1
\end{array}\right]
\end{aligned}
$$

Thus, the vector $\boldsymbol{\rho}_{P}$ to the arbitrary body-fixed point $P$ can be transformed into the inertial coordinate system as shown in (3). Here, the translational shift is taken into account using the vector $\boldsymbol{r}_{C}$.

$$
\boldsymbol{r}_{P}=\boldsymbol{r}_{C}+\mathbf{T} \cdot \boldsymbol{\rho}_{\boldsymbol{P}}
$$

Hence, the current position of the locomotion system is characterized by the six parameters $x_{C}, y_{C}, z_{C}, \alpha, \beta$ and $\gamma$ which are summarized in the vector $\boldsymbol{q}$. However, the locomotion system enables only two degrees of freedom due to four nonholonomic constraints. In order to describe the behavior of the rolling locomotion system these constraints have to be taken into account. Therefore, the velocity of the tensegrity structure is considered. The velocity of an arbitrary body fixed point $P$ is given in (4).

$$
\dot{\boldsymbol{r}}_{P}=\dot{\boldsymbol{r}}_{C}+\frac{\mathrm{d}}{\mathrm{d} t}\left(\mathbf{T} \cdot \boldsymbol{\rho}_{P}\right)
$$

The current contact point in the $\eta-\zeta$-plane of the tensegrity structure is denoted as $Q$ and is specified by $\left.\boldsymbol{\rho}_{Q}=(0,-R \sin (\alpha),-R \cos (\alpha))^{\mathrm{T}}\right)$. Additionally, two specific points attached to the tensegrity structure are considered. The point $T$ represents the point of intersection of the $\xi$-axis and the $x-y$-plane. This point is given by $\boldsymbol{\rho}_{T}=(R / \tan (\beta), 0,0)^{\mathrm{T}}$. The parameter $K$ represents a point on the circumference of the tensegrity structure in $\eta-\zeta$-plane characterized by $\boldsymbol{\rho}_{K}=(0,0,-R)^{\mathrm{T}}$. If $K$ is in contact with the horizontal plane $(\alpha=0)$ the corresponding velocity has to be zero. This issue yields three nonholonomic constraints given in (5). Additionally, the point $T$ does not move perpendicular to the axis given by the current contact point $Q$ and $T\left(v_{\perp}=0\right)$. These issue allows the description of the fourth nonholonomic constraint which is given in (6).

$$
\left.\dot{\boldsymbol{r}}_{P}\right|_{\boldsymbol{\rho}_{P}=\boldsymbol{\rho}_{K}, \alpha=0}=\mathbf{0}
$$

$v_{\perp}=\left(\left.\mathbf{T}_{\gamma}^{\mathrm{T}} \cdot \dot{\boldsymbol{r}}_{P}\right|_{\boldsymbol{\rho}_{P}=\boldsymbol{\rho}_{T}}\right) \cdot\left(\begin{array}{l}0 \\ 1 \\ 0\end{array}\right)=0$

The parameter $\dot{\alpha}$ and $\dot{\beta}$ are chosen as generalized velocities. Considering the nonholonomic constraints
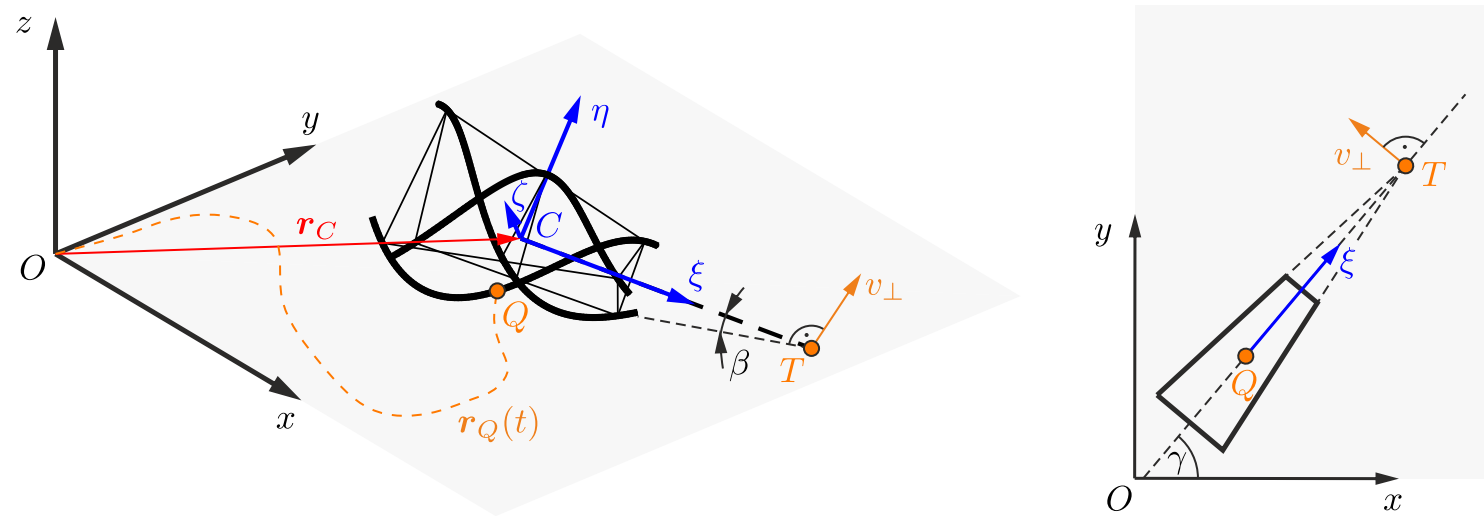

Fig. 3 Mechanical model of the rolling locomotion system in contact with a horizontal plane due to gravity 
in (5) and (6), the parameters $\dot{x}_{C}, \dot{y}_{C}, \dot{z}_{C}$ and $\dot{\gamma}$ can be formulated as shown in (7)-(10).

$$
\begin{aligned}
& \dot{x}_{C}=R \cos ^{2}(\beta) \sin (\gamma) \dot{\alpha}+R \cos (\beta) \cos (\gamma) \dot{\beta} \\
& \dot{y}_{C}=-R \cos ^{2}(\beta) \cos (\gamma) \dot{\alpha}+R \cos (\beta) \sin (\gamma) \dot{\beta} \\
& \dot{z}_{C}=-R \sin (\beta) \dot{\beta} \\
& \dot{\gamma}=\sin (\beta) \dot{\alpha}
\end{aligned}
$$

The generalized velocities represent the actuation of the tensegrity structure. Here, $\dot{\alpha}$ is realized by the movement of internal masses and the angular velocity $\dot{\beta}$ represents the shape change of the structure. Following, the realization of the actuation of the locomotion system is not specified. Therefore, arbitrary actuation parameters $\dot{\alpha}$ and $\dot{\beta}$ are possible.

\section{Locomotion behavior of the rolling locomotion system}

In this section the resulting locomotion due to the actuation parameters $\dot{\alpha}$ and $\dot{\beta}$ is considered for $t \in\left[0, t_{\text {end }}\right]$. Thus, the differential equation formulated in (11) has to be solved taken the nonholonomic constraints (7)-(10) into account. The control parameters $\dot{\alpha}$ and $\dot{\beta}$ are given due to the applied actuation [15-17]. Here, $\mathbf{A}$ represent a matrix $\left(\mathbf{A} \in \mathbb{R}^{6 \times 2}\right)$.

$\boldsymbol{q}=\int_{0}^{t_{\text {end }}}\left(\mathbf{A} \cdot\left(\begin{array}{c}\dot{\alpha} \\ \dot{\beta}\end{array}\right)\right) \mathrm{d} t$

with $\boldsymbol{q}=\left(x_{C}, y_{C}, z_{C}, \alpha, \beta, \gamma\right)^{\mathrm{T}}$

As initial configuration $\boldsymbol{q}_{0}=\boldsymbol{q}(t=0)$ the state $\boldsymbol{q}_{0}=$ $\left(x_{0}, y_{0}, R \cos \left(\beta_{0}\right), 0, \beta_{0}, \gamma_{0}\right)^{\mathrm{T}}$ is chosen. Because of the nonlinear character of (11) a numerical solution using computational support is required. Following, if necessary (11) is solved numerically using a RUNGEKuTTA method. Beside the general locomotion behavior the motion behavior of the cylindrical shaped and conical shaped tensegrity structure is considered.

\subsection{Rectilinear locomotion}

As first approach, the rolling locomotion of the cylindrical tensegrity structure without shape change is considered. Obviously, a rectilinear locomotion will occur. This issue is formulated by $\beta_{0}=0$ and $\dot{\beta}=0$ $(\beta \equiv 0)$. Hence, the nonholonomic constraints formulated in (7)-(10) are simplified to (12)-(15).

$$
\begin{aligned}
& \dot{x}_{C}=R \sin (\gamma) \dot{\alpha} \\
& \dot{y}_{C}=-R \cos (\gamma) \dot{\alpha} \\
& \dot{z}_{C}=0 \\
& \dot{\gamma}=0
\end{aligned}
$$

These constraints are integrable and are transformed into holonomic constraints by integration [18]. This approach yields a linear system of differential equation, which can be solved analytically. The resulting position is given by (16).

$$
\left(\begin{array}{c}
x_{C} \\
y_{C} \\
z_{C} \\
\alpha \\
\beta \\
\gamma
\end{array}\right)=\left(\begin{array}{c}
x_{0}+R \sin \left(\gamma_{0}\right) \alpha \\
y_{0}-R \cos \left(\gamma_{0}\right) \alpha \\
R \\
\int \dot{\alpha} \mathrm{d} t \\
0 \\
\gamma_{0}
\end{array}\right)
$$

Thus, this locomotion mode yields one degree of freedom specified by $\alpha$. The simulation results depicted in Fig. 4 clarify the rectilinear locomotion. The orientation of the uniaxial trajectory is defined by the parameter $\gamma_{0}$. Moreover, the direction of motion (forward locomotion/ backward locomotion) can be controlled by the actuation parameter $\dot{\alpha}$.

\subsection{Circular locomotion}

In this section the locomotion behavior corresponding to the conical shape of the tensegrity structure

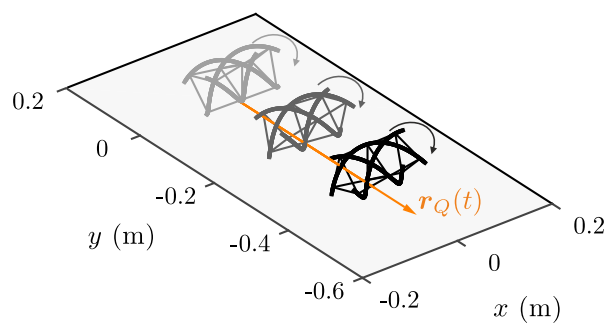

Fig. 4 Simulation of the rectilinear locomotion due to the cylindrical shape of the tensegrity structure for $x_{0}=0, y_{0}=0$, $\gamma_{0}=0$ and $\dot{\alpha}=\pi \mathrm{rad} / \mathrm{s}$ 
characterized by $\beta_{0}$ is considered. Apparently, a circular locomotion occurs due to the outer shape of the tensegrity structure. The corresponding nonholonomic constraints given in (7)-(10) are simplified by $\dot{\beta}=0\left(\beta \equiv \beta_{0}\right)$. This approach is shown in (17)-(20).

$$
\begin{aligned}
& \dot{x}_{C}=R \cos ^{2}\left(\beta_{0}\right) \sin (\gamma) \dot{\alpha} \\
& \dot{y}_{C}=-R \cos ^{2}\left(\beta_{0}\right) \cos (\gamma) \dot{\alpha} \\
& \dot{z}_{C}=0 \\
& \dot{\gamma}=\sin \left(\beta_{0}\right) \dot{\alpha}
\end{aligned}
$$

This locomotion enables only one degree of freedom specified by $\alpha$. Moreover, the constraints listed in (17)-(20) are also integrable. Therefore, these constraints are integrated to holonomic constraints. The resulting linear system of differential equations is solved analytically. This yields (21).

$$
\left(\begin{array}{c}
x_{C} \\
y_{C} \\
z_{C} \\
\alpha \\
\beta \\
\gamma
\end{array}\right)=\left(\begin{array}{c}
x_{0}-R \frac{\cos ^{2}\left(\beta_{0}\right)}{\sin \left(\beta_{0}\right)} \cos \left(\gamma_{0}+\sin \left(\beta_{0}\right) \alpha\right) \\
y_{0}-R \frac{\cos ^{2}\left(\beta_{0}\right)}{\sin \left(\beta_{0}\right)} \sin \left(\gamma_{0}+\sin \left(\beta_{0}\right) \alpha\right) \\
R \cos \left(\beta_{0}\right) \\
\int \dot{\alpha} \mathrm{d} t \\
\beta_{0} \\
\gamma_{0}+\sin \left(\beta_{0}\right) \alpha
\end{array}\right)
$$

In order to clarify the circular locomotion of the conical shaped tensegrity structure, corresponding simulation results are depicted in Fig. 5.

These results confirm that the resulting locomotion trajectory is represented by a circle. The corresponding radius of curvature of the trajectory $\rho_{Q}$ is given by $\left|R / \sin \left(\beta_{0}\right)\right|$. Thus, the cone angle $\beta$ enables the variation of $\rho_{Q}$. The direction of motion (counterclockwise/clockwise) can be controlled by $\dot{\alpha}$ or $\beta$.

\subsection{General locomotion}

The previous locomotion modes are obvious and feature only one degree of freedom. For the realization of a general two-dimensional rolling locomotion system, the shape change of the tensegrity structure has to be taken into account. Hence, the nonholonomic constraints formulated in (7)-(10) cannot be simplified

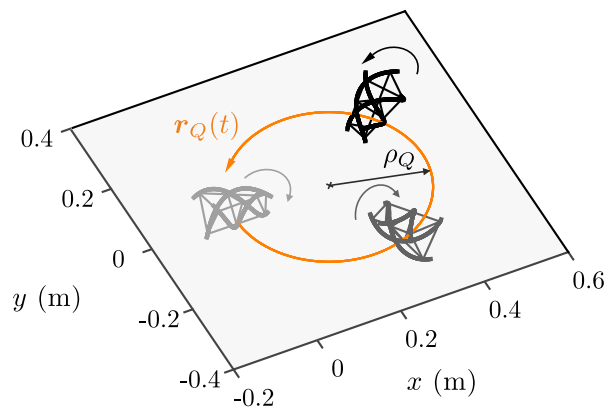

Fig. 5 Simulation of the circular locomotion due to the conical shape of the tensegrity structure for $x_{0}=0, y_{0}=0, \gamma_{0}=0, \beta_{0}=$ $10^{\circ}$ and $\dot{\alpha}=\pi \mathrm{rad} / \mathrm{s}$

or be transformed into holonomic constraints. Due to the nonlinear character of (11) no analytical solution is known. Thus, the nonlinear system of equation given in (11) has to be solved numerically. The resulting rolling locomotion is simulated exemplarily for $x_{0}=0, y_{0}=0, \beta_{0}=0, \gamma_{0}=0, \dot{\alpha}=\pi \mathrm{rad} / \mathrm{s}$ and $\dot{\beta}=$ $0.4 \cos (2 \pi / 5 t) \mathrm{rad} / \mathrm{s}$ for $t \in[0 \mathrm{~s}, 10 \mathrm{~s}]$. The simulation results are depicted in Fig. 6 .

With regard to the simulation results, indeed this locomotion type enables two degrees of freedom specified by $\alpha$ and $\beta$. The combination of the rectilinear locomotion corresponding to the cylindrical shape and the circular locomotion corresponding to the conical shape allows the realization of a general two-dimensional rolling locomotion without tilting sequences. In order to derive an appropriate locomotion strategy to control the locomotion behavior the resulting locomotion trajectories are considered in the following section.

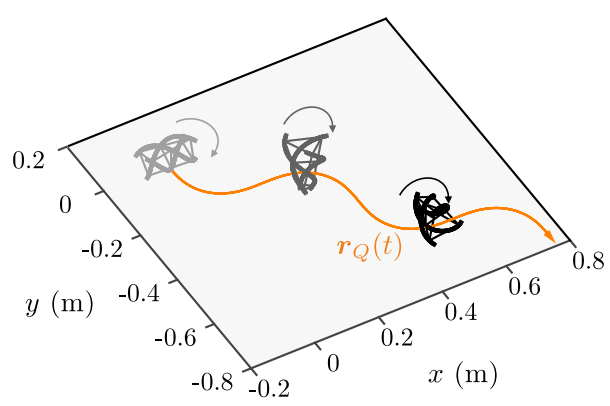

Fig. 6 Simulation of the circular locomotion due to the conical shape of the tensegrity structure with $x_{0}=0, y_{0}=0, \gamma_{0}=0$, $\beta_{0}=0, \dot{\alpha}=\pi \mathrm{rad} / \mathrm{s}$ and $\dot{\beta}=0.4 \cos (2 \pi / 5 t) \mathrm{rad} / \mathrm{s}$ 


\section{Path planning of the locomotion system}

In this section, the various locomotion modes are combined to realize a controllable two-dimensional rolling locomotion. In order to follow a desired a trajectory in the $x-y$-plane a suitable actuation strategy is required. Following, the targeted two-dimensional trajectory $\boldsymbol{\Gamma}$ is formulated respective to the inertial coordinate system $\{x, y, z, O\}$ as parametric equation as shown in (22).

$$
\boldsymbol{\Gamma}=\left(\begin{array}{c}
x_{\Gamma} \\
y_{\Gamma} \\
0
\end{array}\right)
$$

\subsection{Actuation strategy of the locomotion system}

The point $Q$ represents the current contact point in the $\eta-\zeta$-plane of the tensegrity structure. The velocity of $Q$ respective to the inertial coordinate system $\{x, y, z, O\}$ is shown in (23).

$$
\dot{\boldsymbol{r}}_{Q}=\left(\begin{array}{c}
\dot{x}_{Q} \\
\dot{y}_{Q} \\
0
\end{array}\right)=\left(\begin{array}{c}
R \sin (\gamma) \dot{\alpha} \\
-R \cos (\gamma) \dot{\alpha} \\
0
\end{array}\right)
$$

Thus, the trajectory of this point coincide with the horizontal plane. For the following investigations the actuation strategy of the rolling locomotion system is derived to ensure $\boldsymbol{\Gamma}=\boldsymbol{r}_{Q}$. Therefore, these trajectories are evaluated as parametric equations. The properties of the trajectories characterized by the trajectory speed $\dot{s}$ and the corresponding radius of curvature $\rho$. Those characteristic parameters of the trajectory $\Gamma$ are formulated in (24) and (25).

$$
\begin{aligned}
& \dot{s}_{\Gamma}=\sqrt{\dot{x}_{\Gamma}^{2}+\dot{y}_{\Gamma}^{2}} \\
& \rho_{\Gamma}=\frac{{\sqrt{\dot{x}_{\Gamma}^{2}+\dot{y}_{\Gamma}^{2}}}^{3}}{\dot{x}_{\Gamma} \ddot{y}_{\Gamma}-\ddot{x}_{\Gamma} \dot{y}_{\Gamma}}
\end{aligned}
$$

Analogous, the parameters are derived for the trajectory of the point $Q$. This characteristics are simplified applying (23) and (10). This issue yields the Eqs. (26) and (27).

$$
\begin{aligned}
& \dot{s}_{Q}=\sqrt{\dot{x}_{Q}^{2}+\dot{y}_{Q}^{2}}=R|\dot{\alpha}| \\
& \rho_{Q}=\frac{{\sqrt{\dot{x}_{Q}^{2}+\dot{y}_{Q}^{2}}}^{3}}{\dot{x}_{Q} \ddot{y}_{Q}-\ddot{x}_{Q} \dot{y}_{Q}}=\frac{R|\dot{\alpha}|}{\dot{\gamma}}=\frac{R \operatorname{sgn}(\dot{\alpha})}{\sin (\beta)}
\end{aligned}
$$

Obviously, the radius of curvature $\rho_{Q}$ of this trajectory is limited respective to the possible shape change of the system. Therefore, a minimum radius of curvature given by the geometry of the tensegrity structure is defined: $\rho_{Q, \text { min }}=\sqrt{R^{2}+H^{2} / 4}$. Thus, the trajectory $\Gamma$ can be realized if $\left|\rho_{\Gamma}\right| \geq \rho_{Q \text {,min }} \forall t$. Finally, the actuation strategy is derived considering (28) and (29). Here, the sign of the parameter $\dot{\alpha}$ is evaluated with regard to the tangent vector of the trajectory $\boldsymbol{\Gamma}$.

$$
\begin{aligned}
& \dot{\alpha}=-\frac{\dot{s}_{\Gamma}}{R} \cdot\left(\left(\mathbf{T}_{\gamma}^{\mathrm{T}} \cdot\left(\begin{array}{l}
0 \\
1 \\
0
\end{array}\right)\right) \cdot \frac{\dot{\boldsymbol{\Gamma}}}{|\dot{\boldsymbol{\Gamma}}|}\right) \\
& \beta=\arcsin \left(\frac{R \operatorname{sgn}(\dot{\alpha})}{\rho_{\Gamma}}\right)
\end{aligned}
$$

These equations show that the actuation parameter $\dot{\alpha}$ is responsible to guarantee to desired velocity of the trajectory $\dot{s}_{\Gamma}$. The shape change of the tensegrity structure specified by $\beta$ is required to follow the targeted path characterized by the radius of curvature $\rho_{\Gamma}$. Finally, to define the actuation parameter $\dot{\beta}$ the derivative of $\beta$ respective to the time $t$ has to be evaluated $(\dot{\beta}=\mathrm{d} / \mathrm{d} t \beta)$. This issue clarifies that the function $\rho_{\Gamma}$ has to be differentiable to realize the desired trajectory by the rolling locomotion system. If discontinuities exist for $\rho_{\Gamma}$ the locomotion system has to stop $(\dot{\alpha}=0)$ to realize the corresponding radius of curvature. Therefore, the targeted path can be achieved. However, the velocity of the trajectory cannot be enabled.

Utilizing (28) and (29) the trajectory $\Gamma$ can be realized for a suitable initial configuration $\boldsymbol{q}_{0}$ as shown in (30). Moreover, the radius of curvature of the trajectory $\Gamma$ has to fulfill the criterion $\rho_{\Gamma} \geq \rho_{Q, \min } \forall t$. 
Fig. 7 Application of the rolling locomotion system to navigate around an obstacle (grey rectangle) a considered environment with targeted trajectory and various states of the tensegrity structure during the locomotion, b calculated actuation parameter $\dot{\alpha}$, c calculated actuation parameter $\dot{\beta}$

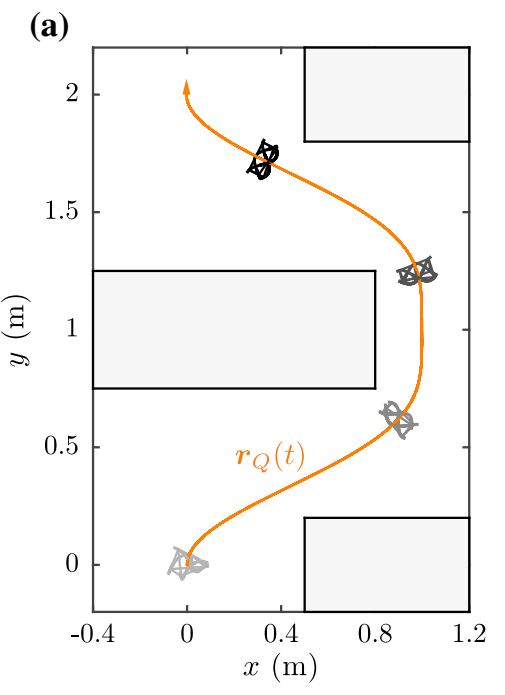

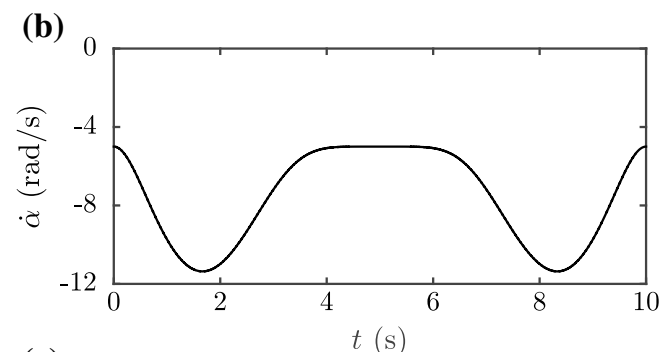

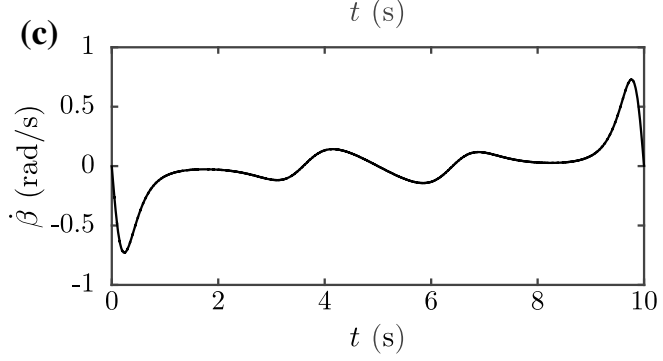

$x_{0}=x_{\Gamma}(t=0)+R \sin \left(\beta_{0}\right) \cos \left(\gamma_{0}\right)$,

$y_{0}=y_{\Gamma}(t=0)+R \sin \left(\beta_{0}\right) \sin \left(\gamma_{0}\right)$,

$z_{0}=R \cos \left(\beta_{0}\right)$

$\beta_{0}=\arcsin \left(\frac{R \operatorname{sgn}(\dot{\alpha}(t=0))}{\rho_{\Gamma}(t=0)}\right)$,

$\gamma_{0}=\arctan \left(\frac{\dot{y}_{\Gamma}(t=0)}{\dot{y}_{\Gamma}(t=0)}\right)-\frac{\pi}{2}$

\subsection{Application example}

In order to highlight this advantageous and feasible actuation strategy the environment depicted in Fig. 7a is considered exemplarily. Because of the obstacles (grey rectangles) the targeted trajectory shown in Fig. $7 \mathrm{a}$ is defined by (31).

$$
\boldsymbol{\Gamma}=\left(\begin{array}{c}
1-0.25\left(1+\cos \left(\frac{2 \pi}{10} t\right)\right)^{2} \\
0.2 t \\
0
\end{array}\right)
$$

An evaluation of the radius of curvature $\rho_{\Gamma}$ confirms that $\left|\rho_{\Gamma}\right| \geq \rho_{Q \text {,min }} \forall t$. Thus, the realization of this trajectory is possible. The initial configuration is chosen considering (30). With regard to (28) and (29) the actuation parameters $\dot{\alpha}$ and $\dot{\beta}$ are calculated. These results are depicted in Fig. 7b, c. Finally, a numerical simulation of the locomotion system implementing the calculated actuation strategy confirms the locomotion on the targeted trajectory $\boldsymbol{\Gamma}$. Various states of the tensegrity structure during the rolling locomotion are depicted in Fig. 7a.

Thus, indeed the considered tensegrity structure with spatially precurved compressed members allows a steerable rolling locomotion on a horizontal plane due to the shape change between a cylindrical geometry and a conical geometry. Furthermore, the presented actuation strategy allows a feasible trajectory control of the rolling locomotion system to navigate through an arbitrarily shaped environment.

\section{Conclusion}

In this work, a tensegrity structure with spatially precurved members inspired by the tensegrity simplex was designed. Due to the variation of the prestress state, the shape of the structure can be influenced. Therefore, a cylindrical shape and a conical shape can be realized. Furthermore, the utilization of internal masses allows the realization of a rolling locomotion. Kinematic analyses considering the nonholonomic constraints show that the cylindrically shaped structure enables a rectilinear locomotion and the conical shape correspond to a circular locomotion trajectory. The application of a shape change during the locomotion enables a rolling locomotion with two degrees of freedom. Thus, each point in the $x-y$-plane can be reached by pure rolling locomotion. Moreover, a feasible actuation strategy to generate a desired path 
with an according trajectory velocity is derived. This approach allows the navigation through an arbitrarily shaped environment. Simulations are evaluated to verify this approach. The results confirm the advantageous properties of the rolling locomotion system and encourage investigations focusing on the development of a prototype and experiments.

Acknowledgements Open Access funding provided by Projekt DEAL. This work is supported by Deutsche Forschungsgemeinschaft (DFG) within the SPP 2100projects ZE714/14-1, BO4114/2-2, BO4114/3-1.

\section{Compliance with ethical standards}

Conflict of interest The authors declare that they have no conflict of interest.

Open Access This article is licensed under a Creative Commons Attribution 4.0 International License, which permits use, sharing, adaptation, distribution and reproduction in any medium or format, as long as you give appropriate credit to the original author(s) and the source, provide a link to the Creative Commons licence, and indicate if changes were made. The images or other third party material in this article are included in the article's Creative Commons licence, unless indicated otherwise in a credit line to the material. If material is not included in the article's Creative Commons licence and your intended use is not permitted by statutory regulation or exceeds the permitted use, you will need to obtain permission directly from the copyright holder. To view a copy of this licence, visit http://creativecommons.org/licenses/by/4.0/.

\section{References}

1. Motro R (2003) Tensegrity: structural systems for the future. Elsevier, Amsterdam

2. Fuller RB (1962) Tensile-integrity structures, U.S. Patent Nr. 3,063,521

3. Snelson K (1965) Continuous tension, discontinuous compression structures. U.S. Patent Nr. 3,169,611

4. Böhm V (2016) Mechanics of tensegrity structures and their application in mobile robotics. Habilitation thesis, Ilmenau University of Technology

5. Skelton R, de Oliveira M (2009) Tensegrity systems. Springer, Berlin

6. Orki O, Shai O, Ayali A, Ben-Hanan U (2011) A model of caterpillar locomotion based on assur tensegrity structures. In: ASME international design engineering technical conferences and computers and information in engineering conference, pp 739-745. https://doi.org/10.1115/ DETC2011-47708
7. Böhm V, Jentzsch A, Kaufhold T, Schneider F, Becker F, Zimmermann K (2012) An approach to locomotion systems based on 3d tensegrity structures with a minimal number of struts. In: ROBOTIK 2012; 7th German conference on robotics, pp 1-6

8. Tietz B, Carnahan R, Bachmann R, Quinn R, SunSpiral V (2013) Tetraspine: robust terrain handling on a tensegrity robot using central pattern generators. In: IEEE/ASME international conference on advanced intelligent mechatronics, pp 261-267. https://doi.org/10.1109/AIM.2013. 6584102

9. Böhm V, Zimmermann K (2013) Vibration-driven mobile robots based on single actuated tensegrity structures. In: IEEE international conference on robotics and automation (ICRA), pp 5475-5480. https://doi.org/10.1109/ICRA. 2013.6631362

10. SunSpiral V, Gorospe G, Bruce J, Iscen A, Korbel G, Milam S, Agogino A, Atkinson D (2013) Tensegrity based probes for planetary exploration: entry, descent and landing (EDL) and surface mobility analysis. Int J Planet Probes 7:13

11. Sabelhaus a, Bruce J, Caluwaerts K, Manovi P, Firoozi R, Dobi S, Agogino A, SunSpiral V (2015) System design and locomotion of SUPERball, an untethered tensegrity robot. In: IEEE international conference on robotics and automation (ICRA), pp 2867-2873. https://doi.org/10.1109/ICRA. 2015.7139590

12. Agogino A, SunSpiral V, Atkinson D (2018) Super Ball Bot-structures for planetary landing and exploration

13. Kaufhold T, Schale F, Böhm V, Zimmermann K (2017) Indoor locomotion experiments of a spherical mobile robot based on a tensegrity structure with curved compressed members. In: IEEE international conference on advanced intelligent mechatronics (AIM), pp 523-528. https://doi. org/10.1109/AIM.2017.8014070

14. Carillo Li e, Schorr P, Kaufhold T, Hernández Rodríguez J, Zentner L, Zimmermann K, Böhm V (2019) Kinematic analysis of the rolling locomotion of mobile robots based on tensegrity structures with spatially curved compressed components. In: Proceedings of the 15th conference on dynamical systems-theory and applications (applicable solutions in non-linear dynamical systems), pp 335-344

15. Murray R (2017) A mathematical introduction to robotic manipulation. CRC Press, Boca Raton

16. Bullo F, Lewis A (2019) Geometric control of mechanical systems: modeling, analysis, and design for simple mechanical control systems, vol 49. Springer, Berlin

17. Murray R, Sastry S (1993) Nonholonomic motion planning: steering using sinusoids. IEEE Trans Autom Control 38(5):700-716

18. Neřmark J, Fufaev N (2004) Dynamics of nonholonomic systems, vol 33. American Mathematical Society, Providence

Publisher's Note Springer Nature remains neutral with regard to jurisdictional claims in published maps and institutional affiliations. 